\title{
Rasgos de personalidad, bienestar y malestar psicológico en usuarios de redes sociales que presentan conductas disruptivas online
}

\author{
Personality traits, well-being and psychological distress in users \\ who present online disruptive behavior
}

María Laura Lupano Perugini ${ }^{1}$ y Alejandro Castro Solano ${ }^{2}$

${ }^{1}$ Doctora en Psicología. Profesora y Licenciada en Piscología. Investigadora Adjunta del Consejo Nacional de Investigaciones Científicas y Técnicas (CONICET), Argentina. Docente de grado y posgrado en la Universidad de Buenos Aires (UBA) y en la Universidad de Palermo (UP).

E-mail:mllupano@hotmail.com

${ }^{2}$ Doctor en Psicología. Investigador Principal del Consejo Nacional de Investigaciones Científicas y Técnicas (CONICET), Argentina. Profesor Adjunto Regular de la Facultad de Psicología de la Universidad de Buenos Aires (UBA). Director del Doctorado en Psicología de la Universidad de Palermo (UP). E-mail: alejandro.castrosolano@gmail.com

El presente trabajo fue realizado mediante los subsidios PIP - CONICET 11220150100381CO: "Hacia una aproximación émica de la psicología positiva. Los rasgos positivos como predictores del funcionamiento óptimo" y con subsidio UBACyT 20020150100037BA: "La evaluación de los rasgos de personalidad positivos. Su relación con los rasgos de personalidad patológicos (DSM-5) y el bienestar psicológico".

Universidad de Palermo.

Universidad de Buenos Aires.

Consejo Nacional de Investigaciones Científicas y Técnicas (CONICET).

Buenos Aires, Argentina.

\section{Resumen}

El presente estudio se propuso investigar perfiles de usuarios de redes sociales que presentan conductas disruptivas online. Para tal fin se diseñó un índice que permite evaluar el nivel en el que se presentan dichas conductas y se analizó la relación entre la intensidad de la conducta disruptiva online con variables sociodemográficas, motivos de usos de redes, variables de personalidad (normales, patológicas y rasgos vinculados a tríada oscura), bienestar y malestar psicoló- gico. Participaron 858 adultos (421 hombres y 437 mujeres) que tenían en promedio 39.16 años $(D E=14.14)$. Para la recolección de datos se administraron: Dark Triad Scale, Big Five Inventory, Inventario para Trastornos de la Personalidad para el DSM-5, Mental Health Continuum y Depression, Anxiety and Stress Scale, y se diseñaron dos escalas: Índice de Conductas Disruptivas Online y Motivos de Utilización de Redes Sociales. En cuanto a las variables sociodemográficas, se halló que los más jóvenes de sexo masculino y que no trabajaban eran quienes presentaban un mayor 
nivel de comportamientos disruptivos online. En cuanto a los motivos de uso de redes, se observó que preferían usarlas con fines meramente exhibicionistas, de ocio o para iniciar nuevas relaciones. Por otro lado, en relación con rasgos de personalidad, aquellos que diferenciaban los perfiles en cuanto a la intensidad de las conductas disruptivas fueron: bajos niveles de responsabilidad y altos niveles de desinhibición, narcisismo y maquiavelismo. Por último, se demostró que presentar este tipo de conductas se asocia con mayor nivel de estrés y menor de bienestar social.

Palabras clave: conductas disruptivas online, motivos de uso de redes sociales, rasgos de personalidad, tríada oscura, bienestar, síntomas.

\section{Abstract}

The present study focused on the analysis of online disruptive behavior that usually occurs among social network users. Individuals who perpetrate such behavior are commonly known as trolls or haters (Cheng, Danescu-Niculescu-Mitzil, \& Leskovec, 2015). In general, they post comments, photos or provocative videos that do not pursue any purpose other than annoying or obtaining pleasure or fun (Brandel, 2007; Phillips, 2011). Recent studies have shown that certain personality traits are associated with the presence of such disruptive behavior. In this research, we studied normal personality traits, psychopathological traits, and dark personality traits in relation to disruptive behavior. The normal personality traits were considered from the classic Five Factor Model (FFM) - Openness to experience, Conscientiousness, Extraversion, Agreeableness, Neuroticism (Costa, \& McCrae, 1985); the psychopathological traits, from the latest version of the DSM - Negative Effect, Detachment, Antagonism, Disinhibition and Psychoticism (DSM-5, American Psychiatric Association, 2013); and the dark personality traits from the so-called Dark Triad model - Narcissism,
Machiavellianism and Psychopathy (Palthus, \& Williams, 2002). This latter model has a growing interest in the international literature, related to dysfunctional Internet use, particularly to social networks.

Therefore, the present study aimed to analyze profiles of social network users who present online disruptive behavior. For this purpose, an index was designed to assess the level of occurrence of such behaviors. First, the relationship between the intensity of online disruptive behavior and sociodemographic variables (i.e., sex, age, studies, occupation and nationality) and motives for social network use (i.e., Relationship maintenance; Passing time and Exhibitionism; Relationship Initiation and Search for companionship) were assessed. Then, the relationships among personality variables (i.e., normal, psychopathological and traits related to the dark triad); well-being (i.e., emotional, social and personal) and psychological distress (i.e., anxiety, stress and depression) were analyzed.

The sample consisted of 858 adults (421 men, $49.1 \%$ and 437 women, $50.9 \%$ ) with a mean age of 39.16 years $(S D=14.14)$. The majority $(67.9 \% ; \mathrm{n}=583)$ lived in Buenos Aires, Argentina). For data collection, the following questionnaires were used: Dark Triad Scale (DTS), Big Five Inventory (BFI), Personality Disorder Inventory (PID-5-BF), the Mental Health Continuum (MHC-SF), and the Depression, Anxiety and Stress Scale (DASS-21). Additionally, two scales were designed: Online Disruptive Behavior Index, and Motives for Social Networks Use. The SPPS 24.0 statistical package was used for data analysis.

Overall, the results are consistent with those reported in the international literature. Regarding sociodemographic variables, it was found that male, younger and unemployed individuals are those who presented higher level of online disruptive behavior. Considering motives for social network use, it was observed that individuals who tend to behave in a dysfunctional manner, preferred to use 
social networks purely for exhibitionism, pastime or relationships initiation. Regarding personality traits, those who differentiated profiles in terms of intensity of disruptive behaviors were: low levels of responsibility and high levels of disinhibition, narcissism and Machiavellianism. Thus, it might be concluded that the overall personality profile tends to be dysfunctional in terms of its characteristics. Finally, it was shown that presenting this type of behavior is associated with higher levels of stress and lower levels of social wellbeing. Therefore, it might be interpreted that although individuals who behave disruptively tend to obtain gratification from injury and deceit to others, this behavior does not result in positive results for them, but rather results in feelings of discomfort from a personal and social point of view.

Keywords: online disruptive behavior, motives for social network use, personality traits, dark triad, mental health, symptoms.

\section{Introducción}

En los últimos años, internet se ha convertido en una extensión de la comunicación cara a cara, dado que se la suele emplear tanto con fines sociales como profesionales o de negocios (Craker y March, 2016). Las estadísticas muestran que a fines de 2019, el 53.6\% de la población mundial accedió diariamente a la red (International Telecommunication Union, 2019) y en especial, hizo uso de las redes sociales como Facebook, Instagram, Twitter, entre otras (Lupano Perugini y Castro Solano, 2019).

Algunos estudios analizan los motivos que llevan a las personas a usar tanto tiempo las redes sociales (Hollenbaugh y Ferris, 2014). En general, las encuestas realizadas dan cuenta de variadas motivaciones de uso, entre las que se enumeran: el mantenimiento de relaciones, pasar el tiempo, desarrollar nuevas relaciones, el entretenimiento, la compañía (Special y Li-Barber, 2012), promocionar actividades laborales/profesionales (e. g., Papacharissi y
Mendelson, 2011) o académicas (e. g., Hew, 2011). En un estudio realizado en Argentina mediante la técnica del análisis factorial exploratorio, se aislaron tres motivos de uso que suelen englobar los hallados en investigaciones previas. El primero se relaciona con usar las redes para estar en contacto con personas conocidas como amigos o familiares (mantenimiento de relaciones personales); el segundo implica un uso orientado al ocio y la necesidad de mostrarse (pasatiempo y exhibicionismo); y el último se relaciona con usar las redes para conocer gente nueva e iniciar nuevas relaciones (inicio de relaciones nuevas) (Lupano Perugini y Castro Solano, 2020).

El uso creciente que se hace de las redes sociales ha generado que, además de los beneficios que implica en cuanto al aumento de posibilidades para socializar y comunicarse, también hayan surgido una serie de comportamientos antisociales online (Williams, 2000). Estos comportamientos provocan en las víctimas las mismas consecuencias psicológicas que cuando se dan cara a cara, como síntomas depresivos, ansiedad social y bajos niveles de autoestima (Kirkaburun, Jonason y Griffiths, 2018; Nicol, 2012).

Existe una serie de comportamientos agresivos online que suelen presentarse entre usuarios de redes sociales y a quienes los perpetran comúnmente se los denomina trolls o haters (Cheng, Danescu-Niculescu-Mitzil y Leskovec, 2015). Pueden actuar solos o con otros, tanto de forma indiscriminada o selectivamente hacia ciertos individuos, grupos sociales, partidos políticos, entidades corporativas, entre otros (Buckels, Trapnel, Andjelovic y Paulhus, 2018). En general, suelen postear comentarios, fotos o videos provocativos que no persiguen otro fin más que molestar u obtener placer o diversión (Brandel, 2007; Phillips, 2011). El anonimato y el sinsentido de las intervenciones distinguen esta conducta online de otros comportamientos abusivos como el ciberbullying, en el que la intención es más clara (Buckels, 
Trapnell y Paulhus, 2014; Lenhardt, 2012). A partir de un análisis de contenido (de comentarios online) realizado por Hardaker (2010) se aislaron cuatro características fundamentales que identifican la conducta troll: el engaño, la agresión, lo disruptivo y el éxito. La combinación de las tres primeras facetas garantiza la última. Hardaker (2010) sugiere que las personas que se involucran en estos comportamientos están motivadas por conseguir éxito en engañar, incomodar y dañar a las personas que son blanco de sus insultos.

Buckels et al. (2014) desarrollaron un índice destinado a la medición de la conducta troll llamado Global Assessment of Internet Trolling (GAIT). Este índice cuenta con una validación realizada para la Argentina (Resett y González Caino, 2019). En el presente estudio, se sigue la línea de razonamiento de Grothe, Staar y Janneck (2016) que sostiene que estas conductas agresivas online pueden recibir diferentes nombres más allá de conducta troll o trolling. Por lo tanto, aquí se adopta la expresión genérica de "conducta disruptiva online" y se propone el diseño de un índice ampliado que focalice en el uso de redes sociales ya que el GAIT abarca todo tipo de sitios de internet incluidos los de juegos online, por lo que no permite un análisis de la presencia de conductas disruptivas exclusivamente en las redes sociales, objetivo de esta investigación.

Investigaciones empíricas recientes (e. g., Buckels et al., 2014, 2018; Craker y March, 2016; March, Grieve, Marrington y Jonason, 2017; Marshall, Ferenczi, Lefringhausen, Hill y Deng, 2018; Sest y March, 2017) han demostrado que determinados rasgos de la personalidad suelen estar asociados con la presencia de este tipo de conductas disruptivas. En general, dichos estudios consideran el modelo de rasgos de personalidad oscura (Dark Triad) (Palthus y Williams, 2002). Este abarca los siguientes rasgos: el narcisismo, el maquiavelismo y la psicopatía. Posteriormente incorpora también el sadismo y da lugar a la constelación denominada Dark
Tetrad (Buckels, Jones y Paulhus, 2013). De acuerdo con los autores, estos rasgos presentan algunos elementos comunes, tales como la insensibilidad interpersonal, la falta de empatía, la ausencia de humildad, la deshonestidad, la manipulación y las creencias en la propia superioridad (Jones y Paulhus, 2017; Stead y Fekken, 2014).

Los estudios realizados hasta el momento han establecido que los rasgos de psicopatía y sadismo son predictores positivos significativos de estas conductas antisociales online. Si bien Buckels et al. (2014) encontraron que el narcisismo y el maquiavelismo correlacionan con este tipo de comportamientos, no hay suficiente evidencia como para considerarlos un predictor de ellos (e. g., Buckels et al., 2014; Craker y March, 2016; March et al., 2017). En relación con el género, se halló que los hombres tienen más probabilidades que las mujeres de perpetrar estos actos disruptivos. Además, la edad correlaciona negativamente con la presencia de este tipo de conductas (e. g., Buckels et al., 2014; Craker y March, 2016).

Por otro lado, si se consideran los rasgos normales de personalidad desde el modelo clásico Five Factor Model (FFM) (Costa y McCrae, 1985), las investigaciones muestran correlaciones negativas con los rasgos de responsabilidad y amabilidad. Por lo tanto, se trata de perfiles de usuarios poco confiables y negligentes (Buckels et al., 2014; Grothe et al., 2016). En la presente investigación se considera, además de los rasgos normales y oscuros de la personalidad, la evaluación de cinco rasgos patológicos (afecto negativo, desapego, antagonismo, desinhibición y psicoticismo) incorporados en la última versión del DSM (DSM-5; American Psychiatric Association, 2013) y que representan versiones patológicas de los incluidos en el FFM (Krueger, Derringer, Markon, Watsony Skodol, 2013). Resulta novedosa su inclusión, ya que hasta el momento no existen muchos trabajos sobre el empleo de redes sociales que los hayan estudiado. En general, estos rasgos 
se vinculan a un uso problemático de internet (Gervasi et al., 2017). Un estudio reciente mostró que los rasgos de antagonismo y desinhibición se asocian mayormente con una utilización de redes sociales con fines meramente exhibicionistas o para conocer gente nueva en comparación con un uso motivado por el mantenimiento de relaciones sociales ya dadas (Lupano Perugini y Castro Solano, 2020).

La mayor parte de las investigaciones sobre este tipo de conductas analizan las consecuencias psicológicas desde el punto de vista de la víctima. En cambio, son pocas las que exploran las consecuencias de perpetrar comportamientos agresivos online (Resett y González Caino, 2019). Algunos estudios lo asocian a un peor funcionamiento psicosocial y baja calidad de vida (Wong, Chan y Cheng, 2014; Fletcher et al., 2014). Además, de acuerdo con algunos autores, los usuarios que presentan estas conductas se caracterizan por bajos niveles de empatía (Sest y March, 2017) y altos niveles de impulsividad disfuncional (March et al., 2017), lo cual puede influir negativamente en un correcto desarrollo de relaciones sociales y, por lo tanto, afectar la percepción de bienestar social (Castro Solano y Cosentino, 2016). En general a estos usuarios no suele importarles el bienestar de los otros y en cambio, se centran en su propio bienestar y gratificación (Grothe et al., 2016).

En virtud de lo expuesto, el presente estudio se propuso investigar perfiles de usuarios de redes sociales que presentaran conductas disruptivas online. Para tal fin, se diseñó un índice que permite evaluar el nivel en el que se presentan dichas conductas. A partir de ello, se analizó la relación entre la intensidad de la conducta disruptiva online con variables sociodemográficas de interés (i. e., sexo, edad, estudios, ocupación y nacionalidad) y motivos de usos de redes (i.e., mantenimiento relaciones, pasatiempo/exhibicionismo, inicio de relaciones). Asimismo, se estudió la relación con variables de personalidad (i. e., normales, patológicas y rasgos vinculados a tríada oscura), bienestar (i. e., emocional, social y personal) y malestar psicológico ( $i$. e., ansiedad, estrés y depresión).

De acuerdo con los antecedentes expuestos, se esperaba encontrar perfiles diferenciales de usuarios de redes sociales que presentaban conductas disruptivas online. Se estimaba que los usuarios que presentaran este tipo de conductas serían en su mayor parte hombres jóvenes. Asimismo, se esperaba encontrar una asociación positiva entre la presencia de conducta disruptiva y ciertos rasgos negativos (e. g., psicopatía) y patológicos (e. g., antagonismo y desinhibición), así como una asociación negativa con los rasgos de responsabilidad y amabilidad. Por último, se estimó que los niveles de conducta disruptiva se asociarían negativamente con los niveles de bienestar social, y positivamente con indicadores de malestar psicológico (e.g., ansiedad, estrés y depresión).

\section{Método}

\section{Participantes}

Se trata de una muestra de conveniencia compuesta por 858 sujetos, 421 hombres $(49.1 \%)$ y 437 mujeres $(50.9 \%)$, que tenían en promedio 39.16 años $(D E=14.14$; mínimo $=18$, máximo $=70)$. Se tuvo como objetivo seleccionar un tamaño muestral con el cual se pudieran hallar correlaciones de, como mínimo, un tamaño del efecto pequeño(Cohen, 1992). Así, se recurrió al cálculo del tamaño muestral, cuyo resultado fue que el mínimo necesario era de 783 participantes (Hulley, Cummings, Browner, Grady y Newman, 2013). El $7.3 \%(n=63)$ de la muestra fueron extranjeros residentes en Argentina. Del total de la muestra, el $67.9 \%(n=583)$ vivían en la Ciudad Autónoma de Buenos Aires y el $26.7 \%(n=229)$, en el conurbano bonaerense. El resto $(n=46,5.4 \%)$ residía en el interior del país. La mayoría trabajaba como empleado $(n=469,54.7 \%)$, el $17.2 \%(n=$ 148) lo hacía por su cuenta y el $5.1 \%$, como 
patrón o empleador $(n=44)$, mientras que 7 participantes $(0.8 \%)$ manifestaron trabajar sin percibir salario por ello. El resto de la muestra $(n=190,22.2 \%)$ eran trabajadores no activos (entre los que se incluyen jubilados, desempleados, amas de casa).

En cuanto al nivel de estudios, el $39.5 \%$ $(n=339)$ refirió tener estudios universitarios o terciarios completos, y el $10.6 \%(n=91)$ tenía estudios de posgrado. Un $31.8 \%(n=$ 273) tenía estudios terciarios o universitarios sin terminar, un $11.3 \%(n=97)$, secundario completo y el resto $(6.8 \% ; n=58)$ tenía secundario incompleto o escolaridad primaria completa. La mayoría de los participantes se percibieron con un nivel socioeconómico medio-bajo $(n=101 ; 11.8 \%)$, medio $(n=$ $544 ; 63.4 \%)$ y medio-alto $(n=178 ; 20.7 \%)$.

\section{Materiales}

\section{Índice de conductas disruptivas online}

Se diseñó un índice compuesto por seis ítems que evalúan un tipo particular de conducta disruptiva que se expresa mayormente en las redes sociales, algunas veces denominada "conducta troll". Se tomaron como base para la redacción de los ítems los propuestos por el Global Assessment of Internet Trolling (GAIT) (Buckels, Trapnell, Delroy y Paulhus, 2014). Para la confección de este índice se hizo foco especialmente en conductas disruptivas presentes en redes sociales, e incluye ítems referidos a la experiencia, el disfrute y la identificación con este tipo de conductas (e. g., "He compartido o enviado publicaciones perturbadoras o controvertidas solo por diversión"). La escala tiene un formato de respuesta Likert en el que los participantes debían indicar la frecuencia de cada una de las conductas online en cinco opciones de respuesta, de 1 (Muy en desacuerdo) a 5 (Muy de acuerdo; ver Anexo). El análisis factorial exploratorio realizado arrojó un único factor. La fiabilidad de la escala fue satisfactoria ( $\alpha=$ .86; ver Resultados).

\section{Motivos de utilización de redes sociales}

Se empleó un instrumento diseñado en un estudio previo (Lupano Perugini y Castro Solano, 2020) para examinar los principales motivos de utilización de redes sociales sobre la base de la escala construida por Sheldon (2008) y adaptada posteriormente por Hollenbaugh y Ferris (2014), Motivations and uses of Facebook. La versión final consta de 31 ítems que se responden según el formato Likert con cinco opciones, de 1 (Muy en desacuerdo) a 5 (Muy de acuerdo). En el estudio mencionado se obtuvieron, mediante la técnica de análisis factorial exploratorio, tres factores: (1) mantenimiento de relaciones personales (e. g., "para comunicarme con los demás", "para estar en contacto con mis amigos", "para buscar personas que hace mucho tiempo que no veo"); (2) pasatiempo y exhibicionismo (e.g, "para llamar la atención", "como pasatiempo", "para saber qué están haciendo los demás"); (3) inicio de relaciones nuevas (e. g., "para encontrar compañía", "para encontrar nuevos amigos", "porque no tengo a nadie con quien estar"). Los tres factores obtenidos tenían altas fiabilidades: mantenimiento de relaciones personales $(\alpha=.75)$, pasatiempo y exhibicionismo $(\alpha=.86)$, inicio de relaciones nuevas $(\alpha=.91)$.

En las pruebas diseñadas y usadas en este estudio, se consideró red social como las diferentes plataformas que permiten a los usuarios crear un perfil público e interactuar con otros usuarios de modo online. Un sitio de red social permite integrar a un nuevo usuario a la red y compartir el contacto con la red de contactos más extensa. En todo momento las personas pueden confirmar o rechazar los pedidos de nuevos contactos y así regular la densidad de su propia red. La red social por excelencia es Facebook.

Además, se emplearon los siguientes instrumentos de evaluación:

1) Dark Triad Scale (DTS) (Jones y Paulhus, 2014; adaptación argentina de Salessi y Omar, 2018). Es un instrumento 
de 24 ítems que evalúa rasgos de la tríada oscura de la personalidad (Paulhus y Williams, 2002). Comprende tres dimensiones: maquiavelismo (e. g., "La mayoría de las personas pueden ser manipuladas"), narcisismo (e. g., "Exijo que me traten con el respeto que merezco") y psicopatía (e. g., "Podría decir cualquier cosa con tal de conseguir lo que quiero"). Cada ítem es valorado sobre una escala tipo Likert de cinco puntos: de 1 (Totalmente en desacuerdo) a 5 (Totalmente de acuerdo). Los estudios de validación realizados en Argentina verificaron, mediante análisis factorial exploratorio y confirmatorio, la estructura de tres factores, que incluso se mantuvo invariante en relación al sexo. Las fiabilidades obtenidas $(\alpha)$ para cada factor fueron también satisfactorias: maquiavelismo: .92, narcisismo: .91 y psicopatía: 89 (Salessi y Omar, 2018).

2) Big Five Inventory (BFI) (John, Donahue y Kentle, 1991; adaptación argentina de Castro Solano y Casullo, 2001). Consiste en un instrumento de 44 ítems que evalúa los cinco grandes rasgos de personalidad (extraversión, agradabilidad, responsabilidad, neuroticismo, apertura a la experiencia). La prueba es un derivado de una prueba de adjetivos de personalidad convertidos en frases cortas para facilitar la comprensión de los elementos del test. Estudios realizados en Argentina verificaron la validez factorial de los instrumentos para población adolescente, población adulta no consultante y población militar (Castro Solano y Casullo, 2001). En todos los casos se obtuvo un modelo de cinco factores que explicaba alrededor del $50 \%$ de la variancia de las puntuaciones. Para esta muestra se obtuvieron valores de fiabilidad $(\alpha)$ adecuados: extraversión: .76; agradabilidad: .82; responsabilidad: .83; neuroticismo: .76; apertura a la experiencia: 85 .

3) Inventario para Trastornos de la Perso- nalidad para el DSM-5, Versión Abreviada Argentina (PID-5-BF). Se utilizó la versión breve del inventario de PID-5 de 220 ítems, el PID-5-BF (Krueger et al., 2013), que consta de 25 ítems que evalúan los cinco rasgos clave de personalidad disfuncional propuestos en la Sección III del DSM-5 (APA, 2013): afectividad negativa, desapego, antagonismo, desinhibición y psicoticismo. Los estudios realizados en población argentina obtuvieron valores de confiabilidad satisfactoria. En cuanto a los estudios de validez realizados, se pudo confirmar la estructura de cinco factores propuesta por los autores. En relación con la validez externa se obtuvo convergencia entre los rasgos patológicos y los rasgos de personalidad normal excepto para la relación psicoticismo/apertura a la experiencia. Asimismo, se encontró que este inventario podía predecir perfiles de alto riesgo para la salud (según los criterios de la OMS) y de bajo bienestar psicológico, tanto hedónico como eudamónico (Góngora y Castro Solano, 2017). En esta muestra, los valores de fiabilidad $(\alpha)$ son los siguientes: afectividad negativa: .80 ; desapego: .79; antagonismo: .75; desinhibición: .85; y psicoticismo: .80.

4) Mental Health Continuum - Short Form (MHC-SF) (Keyes, 2005; adaptación argentina de Lupano Perugini, de la Iglesia y Castro Solano, 2017). Este instrumento de 14 ítems evalúa el grado de: (a) bienestar emocional entendido en términos de afectos positivos y satisfacción con la vida (bienestar hedónico); (b) bienestar social (incluye las facetas de aceptación, actualización, contribución social, coherencia e integración social); y (c) bienestar personal en términos de la teoría de Ryff (1989) (autonomía, control, crecimiento personal, relaciones personales, autoaceptación y propósito). El MHC-SF ha mostrado buenas evidencias de consistencia interna y validez discrimi- 
nante en muestras de adultos de diversos países. Los estudios de validación de este instrumento en Argentina han confirmado la estructura factorial del instrumento y han dado evidencia de una buena validez convergente y consistencia interna en población adulta (Lupano Perugini et al., 2017). La fiabilidad $(\alpha)$ por escala para esta muestra fue: bienestar emocional: .80, bienestar social: .74 y bienestar personal: .77.

5) Depression, Anxiety and Stress Scale (DASS-21) (Lovibond y Lovibond, 1995). Esta prueba reúne tres escalas de autoinforme diseñadas para medir los estados emocionales de depresión, ansiedad y estrés. Cada una de las tres escalas de la DASS-21 contiene siete ítems. La escala de depresión evalúa disforia, desesperanza, devaluación del sentido de la vida, autodesprecio, falta de interés, anhedonia e inercia (e. g., "No podía sentir nada positivo"). La escala de ansiedad evalúa la activación autonómica, la ansiedad situacional y la experiencia subjetiva de afecto ansioso (e.g., "Me siento temboloroso/a"). La escala de estrés es sensible a niveles de activación crónica no específica. Evalúa la dificultad para relajarse y la tendencia a molestarse e irritarse fácilmente (e. g., "Me costó mucho calmarme"). Para el presente estudio, se adaptaron los ítems de la versión chilena de Antúnez y Vinet (2012). Se responde sobre la sintomatología percibida durante la última semana, en una escala Likert con cuatro opciones de respuesta, de 0 (No describe nada de lo que me pasó o senti) a 3 (Sí, esto me pasó mucho o casi siempre). La fiabilidad $(\alpha)$ por escala para esta muestra fue: depresión: .90, ansiedad: .85; estrés: .88.

\section{Procedimiento y análisis de datos}

Los datos fueron recolectados por 25 alumnos de una universidad privada de la ciudad de Buenos Aires que se encontraban realizando una práctica de investigación. Los pasantes conectaron a los participantes mediante su red de contactos personales y los entrevistaron personalmente para que completaran la batería con lápiz y papel. La administración de la batería completa de pruebas demoraba alrededor de una hora. La recolección y carga de datos fue supervisada por un docente investigador. Los participantes fueron voluntarios y no recibieron retribución alguna por su colaboración. Además, según lo establecido por el Consejo Nacional de Investigaciones Científicas y Técnicas en su Resolución D N. ${ }^{\circ} 2857$, se les solicitó el consentimiento informado. El cuadernillo que contenía las encuestas presentaba en su portada una introducción en la que se solicitaba el consentimiento del participante y se aseguraba el anonimato de los datos y su uso exclusivo para investigación. Para participar de la investigación las personas debían ser mayores de edad y contar con algún tipo de acceso a internet.

Para la carga y análisis de los datos se utilizó el paquete estadístico SPSS 24.0. En primer lugar, se depuró la base. No fue necesario eliminar casos, ya que muy pocos contaban con datos perdidos. En cambio, se reemplazó el valor del caso perdido por el valor medio de la variable analizada. Posteriormente, se verificó la estructura factorial del índice diseñado mediante un análisis factorial exploratorio usando el método de máxima verosimilitud (ML). Para establecer si existía asociación entre conductas disruptivas online y motivos de uso de redes sociales se realizaron análisis de correlación de Pearson. En el caso de las variables sociodemográficas, para poder analizar la relación con las variables que fueran discretas, se diseñó una variable dummy tomando el primer cuartil (bajo en conductas disruptivas online) y tercer cuartil (alto en conductas disruptivas online). En esos casos se realizaron cálculos de chi cuadrado. En relación a la edad, se generaron dos grupos (menores de $40 \mathrm{y}$ mayores de 40) teniendo en cuenta las edades comprendidas en la muestra y el uso diferencial que pudieran hacer de las redes. A conti- 
nuación, se estimaron correlaciones de Pearson para estimar la asociación entre las conductas disruptivas online, los rasgos de personalidad (normales, patológicos, y rasgos vinculados a tríada oscura), el malestar y el bienestar psicológico percibido. Por último, se realizaron dos análisis discriminantes con el objetivo de identificar las variables de personalidad, el malestar y el bienestar percibido, que discriminaban al grupo que puntuaba alto en conductas disruptivas online. Se realizó análisis discriminante, ya que la variable dependiente (dummy alto/ bajo en conductas disruptivas online) es de carácter categórico, en tanto que las variables independientes (rasgos de personalidad normales, patológicos, y vinculados a tríada oscura) son continuas. Se trata de una técnica de análisis multivariante que permite explicar la pertenencia de cada individuo a grupos preestablecidos en función de las variables de su perfil, así como cuantificar el peso de cada una de ellas en la discriminación (Tabachnick y Fidell, 2019). Considerando que este estudio pretende una descripción de perfiles de usuarios con conductas disruptivas a partir del análisis de una serie de variables sociodemográficas y psicológicas, se considera que es el análisis más apropiado.

\section{Resultados}

\section{Conductas disruptivas online, características sociodemográficas y motivos de uso de redes sociales}

En primer lugar, se realizó un análisis factorial exploratorio con los ítems del índice de conducta disruptiva online diseñado (test de esfericidad de Bartlett $=1113.50, \mathrm{gl} .=15$, $p<.00001$; Índice Kaiser Meyer Olkin $=.85$ ) del cual se extrajo un factor que explicaba el $59 \%$ de la varianza total. Asimismo, el valor de la fiabilidad fue satisfactorio $(\alpha=.86)$ (Ver detalle del instrumento diseñado en Materiales y Anexo).

Una vez validado el índice, y con el objetivo de identificar los usuarios de redes sociales que tenían conductas disruptivas online, se analizó la relación entre la intensidad de la conducta disruptiva online mediante el índice diseñado $\mathrm{y}$ algunas variables sociodemográficas de interés (sexo, edad, si estudiaba o no, si trabajaba o no y si era extranjero o no). Para analizar la relación con las variables discretas se diseñó una nueva variable dummy tomando el primer cuartil (bajo en conductas disruptivas online) y tercer cuartil (alto en conductas disruptivas online). Se encontraron asociaciones significativas entre las conductas disruptivas online y el sexo de los participantes $\left[\chi^{2}(1,858)=9.03, p=.007\right]$, si estudiaba o no $\left[\chi^{2}(1,858)=8.14, p=.01\right]$ y la edad $[t(1$, $856)=4.29, p<.0001]$. No se encontraron asociaciones entre las conductas disruptivas online y si trabajaba o no $\left[\chi^{2}(1,858)=.23, p\right.$ $>.05]$ y la nacionalidad $[t(1,856)=.096, p$ $>.05]$. Por lo tanto, en la muestra analizada, las conductas disruptivas online aparecen más frecuentemente entre los varones que no estudian y que son más jóvenes. El grupo que puntuaba alto en conductas disruptivas tenía en promedio 35.88 años $(D S=13.77$; ver Tabla 1).

Tabla 1

Nivel de conductas disruptivas online de acuerdo a variables sociodemográficas.

\begin{tabular}{c|c|c} 
& \multicolumn{1}{c}{$\boldsymbol{M}$} & $\boldsymbol{D} \boldsymbol{S}$ \\
\hline Muestra total & 1.35 & .61 \\
Varón & 1.45 & .71 \\
Mujer & 1.25 & .47 \\
$<40$ & 1.42 & .59
\end{tabular}




\begin{tabular}{c|c|c} 
& \multicolumn{1}{c}{$\boldsymbol{M}$} & $\boldsymbol{D} \boldsymbol{D}$ \\
\hline$>40$ & 1.26 & .65 \\
Estudia & 1.32 & .66 \\
No estudia & 1.41 & .60 \\
Trabaja & 1.36 & .62 \\
No trabaja & 1.34 & .55 \\
Extranjero & 1.38 & .57 \\
No extranjero & 1.36 & .61
\end{tabular}

En cuanto a la relación entre conductas disruptivas y uso de redes sociales, se encontraron asociaciones significativas (mantenimiento de relaciones: $\mathrm{r}=.11$, pasatiempo y exhibicionismo: $\mathrm{r}=.39$; inicio de nuevas relaciones: $r=.38 ; p<.01$ en todos los casos). Las correlaciones más altas y significativas se hallaron con los motivos de uso de redes: pasatiempo y exhibicionismo, e inicio de nuevas relaciones. El tamaño del efecto de las correlaciones halladas fue de pequeño ( $\mathrm{rs} \geq$ .10) a moderado ( $\mathrm{rs} \geq .30$ ) (Cohen, 1992).

\section{Conductas disruptivas online y rasgos de personalidad (normales, patológicos, y rasgos vinculados a tríada oscura)}

En segundo lugar, se analizó la relación entre variables de personalidad (normales, patológicas y rasgos vinculados a tríada oscura) y conductas disruptivas online. En la Tabla 2 se puede observar que, de las 14 correlaciones hipotetizadas, se obtuvieron 12 correlaciones significativas. Las correlaciones más elevadas resultaron para el rasgo responsabilidad (correlación negativa), el rasgo patológico desinhibición y los rasgos de la tríada oscura psicopatía y maquiavelismo. El tamaño del efecto de las correlaciones halladas fue de pequeño $(r s \geq .10)$ a moderado $(r s \geq .30)$ (Cohen, 1992).

\section{Tabla 2}

Relación entre conductas disruptivas online y variables de personalidad normales, patológicas y rasgos vinculados a tríada oscura $(N=858)$.

\begin{tabular}{c|c}
\multicolumn{2}{c}{$r$} \\
\hline Rasgos de personalidad & .03 \\
Extraversión & $-.24^{* *}$ \\
Agradabilidad & $-.27^{* *}$ \\
Responsabilidad & $.14^{* *}$ \\
Neuroticismo & -.05 \\
Apertura a la experiencia & \\
Rasgos patológicos & $.11^{* *}$ \\
Afecto negativo & $.10^{*}$ \\
Desapego & $.26^{* *}$ \\
Antagonismo & $.27^{* *}$ \\
Desinhibición & $.23^{* *}$ \\
Psicoticismo & $.27^{* *}$
\end{tabular}




\begin{tabular}{c|c}
\multicolumn{2}{c}{$r$} \\
\hline Tríada oscura & \\
Maquiavelismo & $.34 * *$ \\
Narcisismo & $.24 * *$ \\
Psicopatía & $.39 * *$
\end{tabular}

$* * p<.01 ; * p<.05$

Seguidamente, se intentó identificar las variables personológicas que discriminaban al grupo que puntuaba alto en conductas disruptivas online comparados con el que puntuaba bajo. Para ello, se llevó a cabo un análisis discriminante en el que se introdujeron como variables independientes los cinco rasgos de personalidad normales (neuroticismo, extroversión, apertura a la experiencia, agradabilidad y responsabilidad), las variables vinculadas con los rasgos de personalidad patológicos (antagonismo, afecto negativo, desapego, desinhibición y psicoticismo) y las vinculadas con la tríada oscura (maquiavelismo, narcisismo y psicopatía). Como variable dependiente se incluyó la variable dummy alto/bajo en conductas disruptivas online. Se utilizó la metodología de inclusión por pasos. Se obtuvo una función discriminante que explicaba el $24 \%$ de la varianza (Lambda Wilks $=.80, \chi^{2}=70.59$, $\mathrm{gl}=4, p<.0001)$. Las variables que maximizaban más las diferencias entre ambas agrupaciones eran el narcisismo ( $r$ con la función $=.39$ ), el maquiavelismo ( $r$ con la función $=$ .34 ), la desinhibición ( $r$ con la función $=.27$ ) y la responsabilidad ( $r$ con la función $=-.70$ ). Por lo tanto, en la muestra analizada, el grupo alto en conductas disruptivas online presentaba una baja capacidad empática en las relaciones personales, estaban centrados en sus propios intereses, con presencia de conductas manipulativas, impulsividad, tendencia a la gratificación inmediata y baja capacidad de control de impulsos.

\section{Conductas disruptivas online, malestar y bienestar psicológico percibido}

En tercer lugar, se intentó analizar la relación entre variables vinculadas al bienestar y al malestar psicológico con las conductas disruptivas online. En la Tabla 3 se observa que, de las seis correlaciones hipotetizadas, se obtuvieron cuatro correlaciones significativas. Las correlaciones más elevadas positivas resultaron para el estrés percibido. Las correlaciones más elevadas negativas resultaron para el bienestar personal y el social. El tamaño del efecto es pequeño $(r s \geq .10)$ (Cohen, 1992).

\section{Tabla 3}

Relación entre conductas disruptivas online y variables vinculadas al bienestar y al malestar psicológico $(N=858)$.

\begin{tabular}{c|c} 
& $r$ \\
\hline Malestar psicológico & \\
Depresión & .07 \\
Ansiedad & .08 \\
Estrés & $.15^{* *}$ \\
Bienestar psicológico & \\
Bienestar emocional & $-.10^{* *}$
\end{tabular}




\begin{tabular}{c|c} 
& $r$ \\
\hline Bienestar personal & $-.12 * *$ \\
Bienestar social & $-.08 *$
\end{tabular}

$* * p<.01 ; * p<.05$

Seguidamente, se intentó verificar cuáles de estas variables discriminaban al grupo que puntuaba alto en conductas disruptivas online comparados con el que puntuaba bajo. Para ello, se llevó a cabo un análisis discriminante en el que se introdujeron como variables independientes las tres dimensiones del malestar psicológico percibido (ansiedad, estrés y depresión) y las tres correspondientes al bienestar psicológico (emocional, personal y social). Como variable dependiente se incluyó la variable dummy alto/bajo en conductas disruptivas online. Se utilizó la metodología de inclusión por pasos. Se obtuvo una función discriminante que explicaba el $6 \%$ de la varianza $\left(\right.$ Lambda Wilks $=.94, \chi^{2}=20.02, \mathrm{gl}=$ $2, p<.0001)$. Las variables que maximizaban más las diferencias entre ambas agrupaciones eran el estrés ( $r$ con la función $=-.56)$ y el bienestar social ( $r$ con la función $=.72$ ). Por lo tanto, en la muestra analizada, el grupo alto en conductas disruptivas online se percibía con dificultades para relajarse, con tendencia a molestarse fácilmente, además de no sentir una correcta integración e involucramiento social.

\section{Discusión}

El presente estudio tuvo como objetivo general analizar perfiles de usuarios de redes sociales que presentan conductas disruptivas online. Para tal fin, se diseñó y validó un índice para la medición de este tipo de conductas que, a diferencia del comúnmente utilizado (GAIT; Buckels et al., 2014), permite analizar la presencia de estos comportamientos en usuarios de redes sociales. En general, los resultados obtenidos mediante el empleo del índice diseñado en este trabajo son coincidentes con los relevados en la literatura internacional. Por ejemplo, en cuanto a variables sociodemográficas como el género y la edad, se halló que los más jóvenes y de sexo masculino son quienes presentan un mayor nivel de comportamientos disruptivos, tal como fue hipotetizado y muestran estudios previos (e. g., Buckels et al., 2014; Craker y March, 2016). Además, en la presente investigación se evidenció que aquellos que no estudiaban eran más proclives a comportarse de este modo. Esto lleva a pensar que el ocio puede estar motivando a los más jóvenes a tener estas conductas nocivas de divertimento.

Otro hallazgo interesante es la relación entre la presencia de conductas disruptivas online y los motivos de uso de redes. En consonancia con un estudio previo (Lupano Perugini y Castro Solano, 2020), se observó que las personas que tienden a comportarse de este modo disfuncional prefieren usar las redes con fines meramente exhibicionistas, de ocio o para iniciar nuevas relaciones. Si bien se observó correlación con el uso orientado a mantener contacto con personas ya conocidas, el coeficiente hallado fue menor. En el estudio mencionado también se había observado que estos dos motivos (exhibicionismo e inicio de nuevas relaciones) se asociaban a ciertos rasgos patológicos de la personalidad (antagonismo y desinhibición).

Específicamente en relación con rasgos de personalidad, los resultados obtenidos también se encuentran en consonancia con hallazgos previos y con lo hipotetizado en este estudio. Por un lado, bajos niveles de responsabilidad se asociaron con la presencia de conductas disruptivas online. Esto da cuenta, tal como sostienen otros autores (e. g., Buckels et al., 2014; Grothe et al., 2016) que se trata de perfiles de personalidad negligentes y orientados a la búsqueda del placer y la diversión. 
Además, estos comportamientos antisociales son más frecuentes en sujetos con presencia de rasgos patológicos y negativos, características que, como fuera observado por Gervasi et al. (2017), tienden a vincularse a un uso problemático de internet. Específicamente tienden a ser desinhibidos, impulsivos y egocéntricos, además de presentar características de manipulación social. Cabe destacar que en esta investigación se observó que, si bien todos los rasgos de la tríada oscura correlacionan con las conductas disruptivas, los que hacen diferencia en cuanto a la intensidad de dichas conductas son el narcisismo y el maquiavelismo. En cambio, en los antecedentes consultados, psicopatía y sadismo se describen como los mejores predictores (razón por lo cual se había hipotetizado la relación con psicopatía). Si bien en el presente trabajo no se realizaron análisis de predicción, el resultado obtenido difiere de lo obtenido en dichos estudios (e. g., Buckels et al., 2014; Craker y March, 2016; March et al., 2017).

En síntesis, a partir de los hallazgos se puede concluir que las personas analizadas en este estudio que presentan conductas disruptivas online suelen ser jóvenes de sexo masculino y cuentan con tiempo de ocio. De hecho, se sienten atraídos por usar las redes sociales para pasar el tiempo, mostrarse y/o conocer gente. El perfil global de personalidad tiende a ser disfuncional con la presencia de rasgos narcisistas, conductas impulsivas y manipuladoras. El anonimato que otorgan las redes parece favorecer el desarrollo de estas conductas.

Por último, en la muestra analizada en este estudio se demostró que presentar este tipo de conductas se asocia con mayor nivel de estrés - entendido como dificultad para relajarse y tendencia a molestarse e irritarse fácilmente(Lovibond y Lovibond, 1995) y menor nivel de bienestar social -entendido como el sentido de pertenencia e integración social(Keyes, 2005). De esta manera, se cumplió, en parte, la última hipótesis, que planteaba una relación negativa con el nivel de bienestar social percibido y una relación positiva con todos los indicadores de malestar psicológico. En cambio, en este estudio solo se halló una asociación positiva con los niveles de estrés, pero no con depresión y ansiedad. A partir de esto, puede concluirse que, si bien la intención de quienes cometen estas conductas es obtener gratificación a partir de la injuria y el engaño a otros, esto no redunda en resultados positivos para el perpetrador, sino que más bien da lugar a sentimientos de malestar tanto desde el punto personal como social, tal como ha sido descripto en otros estudios (e. g., Fletcher et al., 2014; Wong et al., 2014). Por ende, tanto víctima como victimario experimentan consecuencias negativas, lo que da cuenta del grado de disfuncionalidad de este tipo de comportamientos. Debe tenerse en cuenta que el porcentaje de varianza explicada en los análisis efectuados es bajo (6\%) lo que afecta la contundencia de las conclusiones extraídas en este estudio.

\section{Comentarios finales}

Se puede destacar, como relevancia del estudio realizado, que este se ha llevado a cabo en el contexto latinoamericano que cuenta con un menor nivel de investigaciones en la temática comparado con otras regiones (i. e., Estados Unidos y países de Europa). Los resultados de esta investigación aportan información acerca de nuevas modalidades de agresión facilitadas por el uso de la tecnología. Resulta relevante estudiar estas nuevas formas de violencia online, ya que los efectos perjudiciales se observan tanto en víctimas como en victimarios. Además, las consecuencias sobre las víctimas suelen ser más nocivas y duraderas que las ocasionadas de forma personal debido, en parte, a la exposición social que sufre la víctima (Park, Nay Kim, 2014). Por otro lado, el presente estudio incluyó el análisis no solo de rasgos normales de personalidad que han sido muy estudiados en relación con el tema, sino también de rasgos patológicos de personalidad y de características negativas desde 
el modelo de la tríada oscura que presenta un creciente interés en la literatura internacional, sobre todo en temáticas vinculadas con un uso disfuncional de internet y en especial, de las redes sociales.

En cuanto a las limitaciones del trabajo realizado, puede considerarse el empleo de medidas de autoinforme para la evaluación de conductas disruptivas online que pueden afectar la validez de los datos. Resultaría enriquecedor complementar con técnicas que permitan analizar directamente los contenidos subidos por las personas a las redes sociales a través de, por ejemplo, métodos que admitan un análisis de las palabras usadas en posteos (e. g., LIWC method; Tausczik y Pennebaker, 2010). Otra limitación se relaciona con que se han evaluado los rasgos oscuros de la personalidad con una medida basada en el modelo original (Dark Triad; Palthus y Williams, 2002) que no incluye al rasgo sadismo, que en investigaciones previas ha mostrado ser uno de los mejores predictores de estas conductas (e. g., Buckels et al., 2014; Craker y March, 2016; March et al., 2017).

Como futuras líneas de trabajo, surge la necesidad de diseñar instrumentos más amplios para evaluar este tipo de conductas, ya que en general suele usarse el GAIT o alguna de sus reformulaciones (como en este estudio). Quizás instrumentos más abarcativos, como el Questionnaire on Counterproductive Online Behavior (QOCB; Grothe et al., 2016), que contempla el análisis de diferentes dimensiones (e.g., provocación, engaño, hostilidad, explotación, entre otras), permita un análisis más comprensivo del fenómeno.

Otro aspecto a tener en cuenta en futuros trabajos es el análisis de perfiles de usuarios de diferentes redes (Facebook, Instagram, Twitter, Snapchat, etcétera), ya que investigaciones previas han demostrado que la preferencia por determinadas redes sociales puede estar determinada por diferencias en los perfiles de personalidad (e.g., Hughes, Rowe, Batey y Lee, 2012; Marshal et al, 2018), con lo cual es dable esperar que los usuarios que presentan conductas disruptivas tengan predilección por perpetrar estos comportamientos en determinados sitios de internet más que en otros.

Por último, próximos estudios podrían analizar el rol particular que cumplen algunas emociones en las conductas que se llevan a cabo en las redes. Por ejemplo, algunos autores han demostrado que la envidia -entendida como una emoción hostil que puede estimular comportamientos agresivos (Smith y Kim, 2007)- puede aumentar por el uso intenso de las redes sociales debido a la tendencia a compararse con otros de mayor estatus (e.g., Burke, Marlow y Lento, 2010; Jordan et al., 2011). Por lo tanto, sería interesante analizar la relación entre esta emoción y el desarrollo de conductas disruptivas online.

\section{Referencias bibliográficas}

American Psychiatric Association (2013). Diagnostic and Statistical Manual of Mental Disorders (5a ed). Arlington, VA: American Psyquiatric Publishing. https://doi.org/10.1176/appi. books.9780890425596

Antúnez, Z. y Vinet, E. V. (2012). Escalas de Depresión, Ansiedad y Estrés (DASS- 21): Validación de la versión abreviada en estudiantes universitarios Chilenos. Terapia Psicológica, 30(3), 49-55. http://doi.org/10.4067/S071848082012000300005

Brandel, M. (2007). Blog trolls and cyberstalkers: How to beat them. Computerworld.

Buckels, E. E., Jones, D. N. y Paulhus, D. L. (2013). Behavioral confirmation of everyday sadism. Psychological Science, 24(11), 2201-2209. http://doi.org/10.1177/0956797613490749

Buckels, E. E., Trapnell, P. y Paulhus, D. L. (2014). Trolls just want to have fun. Personality and Individual Differences, 67, 97-102. http://doi. org/10.1016/j.paid.2014.01.016

Buckels, E. E., Trapnell, P. D., Andjelovic, T. y Paulhus, D. L. (2018). Internet trolling and everyday sadism: Parallel effects on pain perception and moral judgment. Journal of Personality, 1-13. https://doi.org/10.1111/ 
jopy. 12393

Burke, M., Cameron, M. y Lento, T. M. (2010). Social network activity and social well-being. Conference on Human Factors in Computing Systems - Proceedings, 3, 1909-1912. https:// doi.org/10.1145/1753326.1753613

Castro Solano, A. y Casullo, M. M. (2001). Rasgos de personalidad, bienestar psicológico y rendimiento académico en adolescentes argentinos. Interdisciplinaria, 18, 65-85.

Cheng J., Danescu-Niculescu-Mitzil C. y Leskovec J. (2015). Antisocial behavior in online discussion communities. arXiv preprint arXiv:1504.00680

Cohen, J. (1992). A power primer. Psychological Bulletin, 112(1), 155-159. https://doi. org/10.1037/0033-2909.112.1.155

Costa, P. T. y McCrae, R. R. (1985). The NEO Personality Inventory Manual. Odessa, FL: Psychological Assessment Resources.

Craker, N. y March, E. (2016). The dark side of Facebook ${ }^{\circledR}$ : The Dark Tetrad, negative social potency, and trolling behaviours. Personality and Individual Differences, 102, 79-84. http:// doi.org/10.1016/j.paid.2016.06.043

Fletcher, A., Fitzgerald-Yau, N., Jones, R., Allen, E., Viner, R. M. y Bonell, C. (2014). Brief report: cyberbullying perpetration and its associations with socio-demographics, aggressive behaviour at school, and mental health outcomes. Journal of Adolescence, 37(8), 1393-1398. http://doi.org/10.1016/j.adolescence.2014.10.005

Gervasi, A. M., La Marca, L., Lombardo, E. M. C., Mannino, G., Iacolino, C. y Schimmenti, A. (2017). Maladaptive personality traits and internet addiction symptoms among young adults: a study based on the alternative DSM-5 model for personality disorders. Clinical Neuropsychiatry, 14(1), 20-28.

Góngora, V. C. y Castro Solano, A. (2017). Assessment of the Mental Health Continuum in a Sample of Argentinean Adults. Psychology, 8, 303-318. https:// doi.org/10.4236/psych.2017.83018
Grothe M., Staar H. y Janneck, M. (June, 2016). How to treat the troll? An empirical analysis of counterproductive online behavior, personality traits and organizational behavior (Conference Paper).

Hardaker, C. (2010). Trolling in asynchronous computer mediated communication: From user discussions to academic definitions. Journal of Politeness Research, 6(2), 215-242. http://doi. org/10.1515/JPLR.2010.011

Hew, K. F. (2011). Students' and teachers' use of Facebook. Computers in Human Behavior, 27, 662-676. http://doi. org/10.1016/j.chb.2010.11.020

Hollenbaugh, E. E. y Ferris, A. L. (2014). Facebook self-disclosure: Examining the role of traits, social cohesion, and motives. Computers in Human Behavior 30, 50-58.

Hughes, D. J., Rowe, M., Batey, M. y Lee, A. (2012). A tale of two sites: Twitter vs. Facebook and the personality predictors of social media usage. Computers in Human Behavior, 28, 561-569. http://doi. org/10.1016/j.chb.2011.11.001.

Hulley, S. B., Cummings, S. R., Browner, W. S., Grady, D. y Newman, T. B. (2013). Designing clinical research: an epidemiologic approach. 4th ed. Philadelphia, PA: Lippincott Williams y Wilkins.

International Telecommunication Union (2019). Individuals using the Internet, 2005-2019. https://www.itu.int/en/ITU-D/ Statistics/Pages/stat/default.aspx

John, O. P., Donahue, E. M. y Kentle, R. L. (1991). The Big Five Inventory-Versions $4 a$ and 54. Berkeley, CA: University of California, Berkeley, Institute of Personality and Social Research.

Jones, D. N. y Paulhus, D. L. (2014). Introducing the ShortDark Triad (SD3): Abrief measure of Dark Personality traits. Assessment, 21(1), 28-41. http://doi.org/10.1177/1073191113514105

Jones, D. y Paulhus, D. (2017). Duplicity Among the Dark Triad: Three Faces of Deceit. Journal of Personality and Social Psychology. 113. https://doi.org/10.1037/pspp0000139

Jordan, A. H., Monin, B., Dweck, C. S., Lovett, 
B. J., John, O. P. y Gross, J. J. (2011). Misery has more company than people think: Underestimating the prevalence of others' negative emotions. Personality and Social Psychology Bulletin, 37(1), 120-135.

Keyes, C. L. M. (2005). The subjective well-being of America's youth: toward a comprehensive assessment. The Journal of Adolescence and Family Health., 4, 3-11.

Kircaburun, K., Jonason, P. K. y Griffiths, M. D. (2018). The Dark Tetrad traits and problematic social media use: The mediating role of cyberbullying and cyberstalking. Personality and Individual Differences, 135, 264-269.

Krueger, R. F., Derringer, J., Markon, K. E., Watson, D. y Skodol, A. E. (2013). The Personality Inventory for DSM-5-Brief Form (PID-5-BF)—Adult. Massachusetts: American Psychiatric Association

Lenhart, A. (2012). Teens, smartphones y texting. http://www.pewinternet.org/2012/03/19/ teens-smartphones-texting/

Lovibond, S. H. y Lovibond, P. F. (1995). Manual for the Depression Anxiety y Stress Scales (2nd Ed.). Sydney: Psychology Foundation.

Castro Solano, A. y Cosentino, A. (2016). The relationships between character strengths and life fulfillment in the view of lay-people in Argentina. Interdisciplinaria, 33(1), 65-80. https://doi.org/10.16888/interd.2016.33.1.4

Lupano Perugini, M. L., de la Iglesia, G., Castro Solano, A. y Keyes, C. L. M. (2017). The Mental Health Continuum-Short Form (MHC$\mathrm{SF})$ in the Argentinean context: confirmatory factor analysis and measurement invariance. Europe's Journal of Psychology, 13, 93-108. https://doi.org/10.5964/ejop.v13i1.1163

Lupano Perugini, M. L. y Castro Solano, A. (2019). Características psicológicas diferenciales entre usuarios de redes sociales de alta exposición vs. no usuarios. Acta Psiquiátrica y Psicológica de América Latina, 65(1), 5-16.

Lupano Perugini, M. L. y Castro Solano, A. (2020). Normal and maladaptive person- ality traits as predictors of motives for social media use and its effects on wellbeing. Psychological Reports. https://doi. org/10.1177/0033294120922495

March, E., Grieve, R., Marrington, J. y Jonason, P. K. (2017). Trolling on Tinder ${ }^{\circledR}$ (and other dating apps): Examining the role of the Dark Tetrad and impulsivity. Personality and Individual Differences, 110, 139-143. http://doi. org/10.1016/j.paid.2017.01.025

Marshall, T. C., Ferenczi, N., Lefringhausen, K., Hill, S. y Deng, J. (2018, December 13). Intellectual, Narcissistic, or Machiavellian? How Twitter Users Differ From Facebook-Only Users, Why They Use Twitter, and What They Tweet About. Psychology of Popular Media Culture. Advance online publication. http:// doi.org/10.1037/ppm0000209

Nicol, S. (2012). Cyber-bullying and trolling. Youth Studies Australia, 31(4), 3-4.

Papacharissi, Z. y Mendelson, A. (2011). Toward a new(er) sociability: Uses, gratifications and social capital on Facebook. En S. Papathanassopoulos (Ed.), Media perspectives for the 21st century (pp. 212-230). NY: Routledge.

Park, S., Na, E. y Kim, E. (2014). The relationship between online activities, netiquette and cyberbullying. Children and Youth Services Review, 42, 74-81. http://doi.org/10.1016/j. childyouth.2014.04.002

Palthus, D. L. y Williams, K. M. (2002). The Dark Triad of personality: Narcissism, Machiavellianism, and psychopathy. Journal of Research in Personality, 36, 556-563. http:// doi.org/10.1016/S0092-6566(02)00505-6

Phillips, W. (2011). LOLing at tragedy: Facebook trolls, memorial pages and resistance to grief online. First Monday, 16(12), 1-14. http://doi. org/10.5210/fm.v16i12.3168

Resett, S. y González Caino, P. C., (2019). Propiedades psicométricas de un Cuestionario de Trolling en una muestra argentina. Revista Argentina de Ciencias del Comportamiento, 11(1), 48-57

Ryff, C. D. (1989). Happiness is everything, or is it? Explorations on the meaning 
of psychological well-being. Journal of Personality and Social Psychology, 57, 1069-1081. http://doi.org/10.1037/00223514.57.6.1069

Salessi, S. y Omar, A. (2018). Psychometric properties of a scale to measure the dark side of personality. Estudos de Psicologia (Campinas), 35(2), 159-170. http://doi.org/10.1590/1982$\underline{02752018000200005}$

Sest, N. y March, E. (2017). Constructing the cyber-troll: Psychopathy, sadism, and empathy. Personality and Individual Differences, 119, 69-72. http://doi.org/10.1016/j. paid.2017.06.038

Sheldon, P. (2008). Student favorite: Facebook and motives for its use. Southwestern Mass Communication Journal, 23(2), 39-53.

Smith, R. H. y Kim, S. H. (2007). Comprehending envy. Psychological Bulletin, 133(1), 46-64. http://doi.org/10.1037/0033-2909.133.1.46

Special, W. P. y Li-Barber, K. T. (2012). Self-disclosure and student satisfaction with Facebook. Computers in Human Behavior, 28(2), 624-630. https://doi. org/10.1016/j.chb.2011.11.008

Stead, R. y Fekken, G. (2014). Agreeableness at the core of the dark triad of personality. Individual Differences Research, 12(4A), 131-141. http://en.buaa.findplus.cn/?h=articlesydb $=\mathrm{a}-$ 9hyan=100210820r

Tabachnick, B. G. y Fidell, L. S. (2019). Using multivariate statistics. Seventh edition. Boston: Pearson Education.

Tausczik, Y. R. y Pennebaker, J. W. (2010). The psychological meaning of words: LIWC and computerized text analysis methods. Journal of Language and Social Psychology, 29, 24-54.

Williams, M. (2000). Virtually criminal: Discourse, deviance and anxiety within virtual communities. International Review of Law Computers y Technology, 14(1), 95-104. http:// doi.org/10.1080/13600860054935

Wong, D. S., Chan, H. C. O. y Cheng, C. H. (2014). Cyberbullying perpetration and victimization among adolescents in Hong Kong. Children and Youth Services Review, 36, 133-140. http:// doi.org/10.1016/j.childyouth.2013.11.006

Recibido: 31 de julio de 2019 Aceptado: 20 de enero de 2021 


\section{Anexo}

Índice de conductas disruptivas online

Indica el grado de acuerdo con las siguientes afirmaciones acerca de acciones que pueden hacerse en las redes sociales.

\begin{tabular}{|c|l|l|l|l|l|}
\hline & $\begin{array}{c}\text { Muy en } \\
\text { desacuerdo }\end{array}$ & $\begin{array}{c}\text { En } \\
\text { desacuerdo }\end{array}$ & $\begin{array}{c}\text { Ni de acuerdo } \\
\text { / ni en } \\
\text { desacuerdo }\end{array}$ & $\begin{array}{c}\text { De } \\
\text { acuerdo }\end{array}$ & $\begin{array}{c}\text { Muy de } \\
\text { acuerdo }\end{array}$ \\
\hline $\begin{array}{c}\text { 1. He compartido o } \\
\text { enviado publicaciones } \\
\text { perturbadoras o contro- } \\
\text { vertidas solo por diver- } \\
\text { sión. }\end{array}$ & & & & & \\
\hline $\begin{array}{c}\text { 2. Me gusta/divierte } \\
\text { molestar en las secciones } \\
\text { de comentarios. }\end{array}$ & & & & & \\
\hline $\begin{array}{c}\text { 3. Cuanto más lindo y } \\
\text { puro es algo, genera más } \\
\text { satisfacción corromperlo. }\end{array}$ & & & & & \\
\hline $\begin{array}{c}\text { 4. Suelo subir comenta- } \\
\text { rios que generan contro- } \\
\text { versia. }\end{array}$ & & & & & \\
\hline $\begin{array}{c}\text { 5. Suelo hacer comen- } \\
\text { tarios que sirven para } \\
\text { desviar la atención. }\end{array}$ & & & & & \\
\hline $\begin{array}{c}\text { 6. Suelo hacer comen- } \\
\text { tarios molestos en los } \\
\text { perfiles de gente recono- } \\
\text { cida/famosa. }\end{array}$ & & & & & \\
\hline
\end{tabular}

